\title{
An Improved Link Adaptation Scheme for High Speed Downlink Packet Access
}

\author{
Hassaan Touheed, Atta Ul Quddus, Rahim Tafazolli \\ Centre for Communication Systems Research \\ University of Surrey, Guildford GU2 7XH, United Kingdom
}

\begin{abstract}
Adaptive Modulation and Coding (AMC) is one of the important features of High-Speed Downlink Packet Access (HSDPA). The link adaptation is done at the Node-B on the basis of Channel Quality Indicator (CQI) reports sent by the User Equipment (UE). However the performance of AMC scheme is degraded due to the large delay between computation of CQI report at $U E$ and the corresponding Transport Format and Resource Indicator (TFRI) selection at the Node-B. An improved link adaptation approach, named CQI averaging herein, is proposed in this paper. In this approach, a number of consecutively received CQI reports from UE are mapped to equivalent Signal to Interference Ratio (SIR) values and passed through a moving average filter. The resulting average is used to derive the CQI index (named Average CQI herein) to be followed at Node-B transmitter. This approach provides a significant gain, especially for medium vehicular speeds. The gain is more pronounced for channels that provide less multipath diversity. Simulation results are used to relate desirable filter length of the moving average filter with the characteristics of the radio channel. Subsequently, an algorithm for adaptation of filter length based on the statistics of CQI reports is proposed.
\end{abstract}

Index Terms - WCDMA; HSDPA; CQI; AMC; CQI averaging.

\section{INTRODUCTION}

HSDPA was included in 3GPP's (3rd Generation Partnership Project) Release-5 specifications [1] and has been the front-line technology within the 3GPP. HSDPA has been termed as $3.5 \mathrm{G}$ of mobile communications and provides the path of mid-term evolution of technology and standardisation. Some key new features introduced by HSDPA are Layer 1 retransmissions, fast scheduling and adaptive modulation and coding; these features enable a physical-layer throughput that makes HSDPA a strong candidate for the provision of broadband wireless access. HSDPA performs AMC by using either QPSK or 16-QAM modulation, combined with variable rate Turbo coding. A base $1 / 3$ rate Turbo coded data are punctured or repeated to achieve various coding rates. Based upon instantaneous channel conditions, a combination (known as TFRI) of QPSK or 16-QAM, code rate and number of physical channels (up to a maximum of 15) is used. For this purpose, CQI tables have been defined for different UE capability categories [4]; each table lists various TFRIs. UE categories are defined according to the maximum number of physical channels they can receive $(5,10$ or 15$)$ and the modulation schemes they can support (QPSK only or both QPSK and 16-QAM). SIR estimate at the UE is mapped to a CQI index according to an SIR to CQI mapping relationship. A few possible SIR to CQI index mapping relationships are given in each of [5-8]. The CQI index points to the highest TFRI in the relevant table that can be used while maintaining a certain quality of service, such as a Block Error Rate (BLER) of $10 \%$. The index is transmitted in the uplink on control channel and is used by Node-B to select the TFRI for next transport block to be transmitted. The Node-B Scheduler may or may not schedule a certain user during a particular Transmission Time Interval (TTI), which is a $2 \mathrm{~ms}$ ( 3 time-slot) period during which different users can be code-multiplexed.

The UE transmits CQI reports in the uplink with a variable reporting period from $2 \mathrm{~ms}$ to $160 \mathrm{~ms}$ [9]. The degradation in HSDPA system throughput with longer reporting periods was studied in [10], wherein it was also shown through simulations that the degradation is more pronounced at higher UE speed. However, even with shortest CQI reporting period of $2 \mathrm{~ms}$, there is a significant delay between CQI estimation at the UE and the corresponding TFRI selection at Node-B. This delay is approximately $10 \mathrm{~ms}[9,11]$. At medium to high mobility, the radio channel and hence the received SIR changes rapidly. Therefore, the validity of a CQI index is significantly reduced at the time it is used. This phenomenon degrades the link performance. It is shown in this paper that the performance of $\mathrm{AMC}$ can be improved through the use of a moving average filter at Node-B. This process takes CQI reports from UE, maps them to received SIR values, performs moving average filtering on these SIR values and maps back the average SIR to a CQI index. The TFRI corresponding to the CQI index thus derived is followed at the Node-B transmitter. It is shown through simulations that the proposed scheme can provide gains in HSDPA throughput that in some cases are in excess of $80 \%$. Furthermore, it is argued that the appropriate order of the moving average filter, i.e., the appropriate averaging window size depends on the characteristics of the fading channel (mainly the Doppler). In order to adapt the averaging window size for optimum performance, the downlink fading channel needs to be characterized at the Node-B. Towards this objective, a mobile velocity estimation method using the properties of auto-covariance of the uplink signal power was presented in [13]. In another approach [14], the authors used the mean and LCR of CQI reports to characterize the fading environment of the mobile. However, the model presented in [14] does not take intra-cell interference into consideration. In our work, we showed that the standard deviation of CQI reports can also be used to characterize the fading channel for selection of suitable CQI averaging window size. A simple algorithm based on the measurement of the standard deviation of CQI indices reported by UE is proposed for adaptation of averaging window size. 
The paper is organized as follows: Section II presents the signal model and receiver architecture, section III describes the CQI averaging process, section IV discusses the simulations results and presents an algorithm for averaging window size selection. Section V concludes the paper.

\section{SIGNAL MODEL}

We consider a WCDMA downlink with $K$ different code channels and a multipath channel with delay spread of $L$ chip intervals. The complex baseband representation of the received multi-user chip sequence is given as:

$$
\mathbf{r}=\sum_{k=1}^{K} \mathbf{H S}_{k} \mathbf{A}_{k} \mathbf{d}_{k}+\mathbf{n}
$$

Vector $\mathbf{r}$ has a length of $N_{c}$, the number of chips in the observation window. $\mathbf{S}_{\boldsymbol{k}}$ is the channelization code matrix for channel $k$. It is a block diagonal matrix that repeats product of Walsh spreading codes and pseudo-random scrambling codes in successive columns for the number of symbols used in the observation window. Dimensions of $\mathbf{S}_{k}$ are $N_{c}$ by $N_{c} / F_{k}$, where $F_{k}$ is the spreading factor for $k$ th channel. Amplitude matrix $\mathbf{A}_{k}$ is a diagonal matrix for $k$ th code channel, having an order equal to the number of symbols in the observation window, i.e., $N_{c} / F_{k}$. Vector $\mathbf{d}_{k}$ is the vector of length $N_{c} / F_{k}$ containing $k$ th channel symbols at the output of modulator and $\mathbf{n}$ is the vector of length $N_{c}$ containing additive noise, which represents thermal noise and interference from other base stations both modelled as White Gaussian noise. The channel matrix $\mathbf{H}$ is sparse and contains coefficients of an $N_{p}$ path fading channel in each row and the row contains leading zeros to represent chip delays for successive chips. Rows of $\mathbf{H}$ also contain zero padding at the end. The dimensions of $\mathbf{H}$ are $N_{c}$ by $\left(N_{c}+L-1\right)$. One sample is taken per-chip. Therefore, the path delays are rounded off to the nearest whole number of chip periods.

The total transmit power of the desired base station is kept constant at unity. A part of this power is allocated to the desired user, a part to Common Pilot Channel (CPICH) and the rest to Orthogonal Channel Noise Simulator (OCNS), a mechanism for modelling intra-cell interfering users [12].

At the receiving end, a Rake receiver is used that comprises of a number of 'fingers', each of which descrambles the received signal and correlates the descrambled signal with channelization code of the desired channel. Finger delays are adjusted to individual channel tap delays. Maximal ratio combining of the fingers follows. Therefore, the signal presented to the demodulator is:

$$
\mathbf{y}=\mathbf{S}_{k}^{H} \mathbf{H}^{H} \mathbf{r}
$$

Here $(.)^{\mathrm{H}}$ represents Hermitian transpose.

\section{CQI AVERAGING}

The UE transmits CQI reports (in the form of indices) in the uplink with a certain reporting frequency. A report can be generated every $2 \mathrm{~ms}, 10 \mathrm{~ms}, 20 \mathrm{~ms}, 40 \mathrm{~ms}, 80 \mathrm{~ms}$ or $160 \mathrm{~ms}$ [9]. For the work presented in this paper, shortest reporting period of $2 \mathrm{~ms}$ is used. CQI indices are derived from the SIR estimates through the relationship proposed jointly by Motorola and Ericsson [5]. This relationship is reproduced in (3).

$$
\mathrm{CQI}^{i}=4.5+\mathrm{SIR}_{d B}^{i}
$$

Here $\mathrm{CQI}^{i}$ is the $i$ th reported index corresponding to the $i$ th SIR estimate in $d B$, i.e., $\operatorname{SIR}_{d B}^{i}$. When a UE receives a packet on the High Speed Downlink Shared Channel (HS-DSCH), it responds with an ACK/NACK and CQI report, transmitted over the uplink High-Speed Dedicated Physical Control Channel (HS-DPCCH) during one TTI. UE response is synchronous such that it starts transmitting this ACK/NACK and CQI frame within 256 chips delay after 7.5 time slots time period from the end of HS-DSCH transport block (TB) transmission [9]. The transmission of ACK/NACK and CQI takes 3 time slots. If the UE is scheduled for the next TTI and signaled over downlink High-Speed Shared Control Channel (HS-SCCH), the start of corresponding HS-DSCH TB takes 2 time slots after the start of HS-SCCH TB; thereby the start of HS-DSCH TB takes place approximately 4.5 time slots after the end of HS-DPCCH transmission carrying the CQI [9]. The total delay between the generation of a CQI report and its application at the Node- $\mathrm{B}$, therefore, is approximately $7.5+3$ $+4.5=15$ time slots (equal to 5 TTIs or $10 \mathrm{~ms}$ ). If the mobile is moving at a relatively fast speed, then the channel variation is rapid enough to make the CQI estimate significantly inaccurate after $10 \mathrm{~ms}$. In other words, high Doppler shift would cause received SIR to significantly change over the 5TTI period. It is intuitively obvious that if the selection of a TFRI is made on the basis of a relatively longer-term average SIR than a 1 TTI average, better performance can be achieved. However excessive averaging at the UE is not feasible as it will not meet the CQI reporting variance and BLER requirements specified by $3 \mathrm{GPP}$ [12]. The CQI reports that are available to Node-B can be translated into the quantized versions of SIR estimates using (3). It is therefore logical to do a longer-term SIR averaging at the Node-B. This is done by mapping the received CQI indices to the equivalent SIR values, averaging the SIR values and finally deriving an average CQI index from SIR to CQI mapping relationship. This average CQI index is used for selection of TFRI instead of the last received CQI index. For the $i$ th received CQI index, the equivalent $\operatorname{SIR}\left(\mathrm{SIR}^{i}\right)$ is obtained from (3) as:

$$
\begin{gathered}
\operatorname{SIR}_{d B}^{i}=-4.5+\mathrm{CQI}^{i} \\
\operatorname{SIR}^{i}=100^{\left(\operatorname{SIR}_{d B}^{i} / 10\right)} \\
\operatorname{SIR}_{a v}^{i}=\left(1 / N \sum_{m=0}^{N-1} \operatorname{SIR}^{i-m}\right. \\
\operatorname{SIR}_{a v, d B}^{i}=10 * \log _{10}\left(\operatorname{SIR}_{a v}^{i}\right) \\
\operatorname{CQI}_{a v}^{i}=4.5+\operatorname{SIR}_{a v, d B}^{i}
\end{gathered}
$$

Here the subscript $d B$ represents values in $\mathrm{dB}$ as opposed to linear values; the subscript $a v$ represents averaged value and $N$ is the averaging window length. 
The scheme presented above does not require any change in the air-interface and requires only a small increase in complexity that can be easily afforded at the Node-B. Terminal behaviour does not require any change.

\section{SIMULATION RESULTS}

Simulation parameters are listed in Table 1. Ratio of energy per chip of HS-DSCH to total transmit power of own base station $\left(\mathrm{E}_{\mathrm{c}} / \mathrm{I}_{\mathrm{or}}\right)$ is chosen to be $-6 \mathrm{~dB}$ whereas the geometry factor (ratio of power received from own base station to the sum of powers received from interfering base stations) of $10 \mathrm{~dB}$ is used. Both the values are in line with 3GPP test recommendations [12]. Channel estimation is performed on $\mathrm{CPICH}$. Path delay estimation is ideal whereas coefficients are estimated over one time slot. UE Category 5, 6 is selected so that there is a continuous transmission to the UE. A variety of ITU radio channels [12] are used. SIR estimation is performed over CPICH and averaged over 3 time slots. HS-DSCH SIR is estimated from CPICH SIR using ratio of the spreading factors and assigned powers to both channels. SIR estimation method used is explained in [15].

TABLE I. SIMULATION PARAMETERS

\begin{tabular}{ll}
\hline Chip Rate & 3.84 Mcps \\
Sampling Rate & 1 Sample per Chip \\
Channel Estimation & Tap Delay: Perfect \\
& Tap gain: Estimated (CPICH) \\
Number of Transmit Antennas & 1 \\
Number of Receive Antennas & 1 \\
Number of Rake Fingers & Equal to channel taps \\
$\mathrm{E}_{\mathrm{c}} \mathrm{I}_{\text {or }}$ CPICH & $-10 \mathrm{~dB}$ \\
$\mathrm{E}_{\mathrm{c}} \mathrm{I}_{\mathrm{or}}$ HS-DSCH & $-6 \mathrm{~dB}$ \\
Geometry Factor & $10 \mathrm{~dB}$ \\
No. of HARQ Processes & 6 \\
Inter-TTI Distance (TTI) & 1 \\
No. of Code Blocks per TTI & 1 \\
Max. No. of Retransmissions & 4 \\
No. of HS-DSCH & Up to 5 \\
UE Category & 5,6 \\
CQI Feedback Rate & Once every TTI (2 ms) \\
Feedback Error Rate & 0 \\
\hline
\end{tabular}

\section{A. Effect of Averaging Window Length}

In this sub-section, the effect of averaging window length for various fading channels [12] is presented. Fig. 1 shows the percentage change in HSDPA throughput against various averaging window sizes (or averaging filter lengths) for different fading channels with medium to high mobility whereas Fig. 2 presents corresponding results in low mobility scenarios. The window size of 1 represents the normal operation without CQI averaging. Percentage change in HSDPA link throughput is plotted instead of the throughput itself so as to conveniently present of the effect of CQI averaging for various fading channels in one figure. Maximum averaging window sizes of 20 and 36 are used for low and high mobility cases respectively.

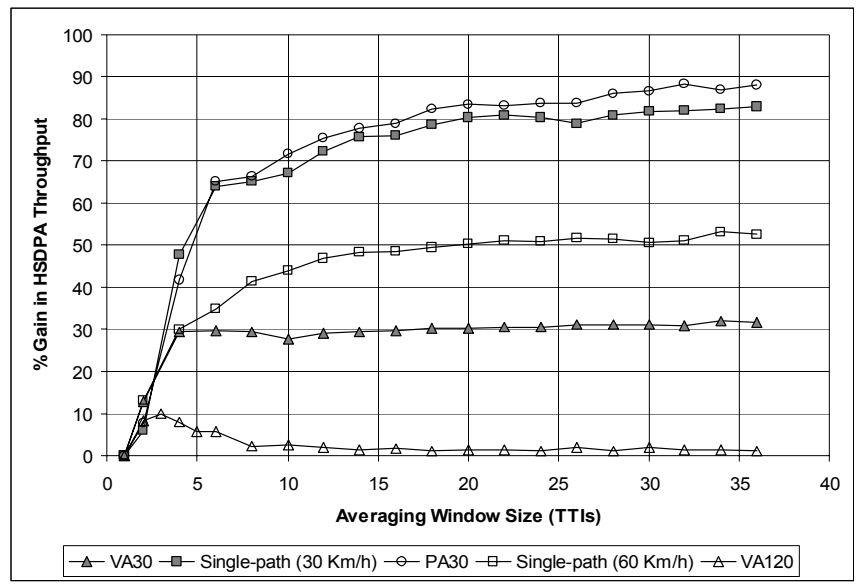

Figure 1: Effect of Averaging Window Size (Medium to High Mobility)

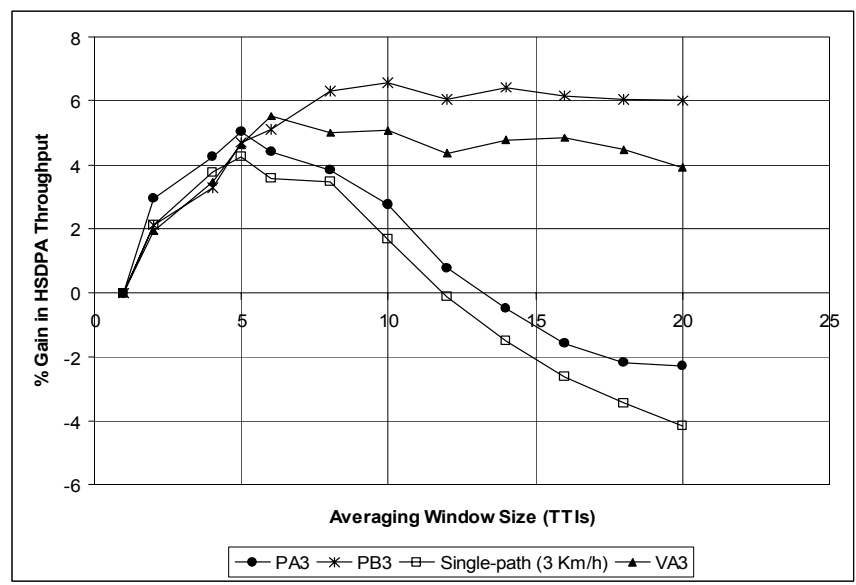

Figure 2: Effect of Averaging Window Size (Low Mobility)

It is clear from Fig. 1 and Fig. 2 that the largest gains are achieved for the Pedestrian-A channel at $30 \mathrm{~km} / \mathrm{h}$ and singlepath channel at $30 \mathrm{~km} / \mathrm{h}$. These peak gains are approximately $86 \%$ and $88 \%$. Single-path channel at $60 \mathrm{~km} / \mathrm{h}$ provides a peak gain of approximately $53 \%$. The peak gains for Vehicular-A channel at $30 \mathrm{~km} / \mathrm{h}$ (VA30), Vehicular-A channel at $120 \mathrm{~km} / \mathrm{h}$ (VA120), Vehicular-A channel at $3 \mathrm{~km} / \mathrm{h}$ (VA3), Pedestrian-A Channel at $3 \mathrm{~km} / \mathrm{h}$ (PA3), Pedestrian-B Channel at $3 \mathrm{~km} / \mathrm{h}$ (PB3) and single-path channel at $3 \mathrm{~km} / \mathrm{h}$ are approximately $32 \%, 10 \%, 6 \%, 5 \%, 7 \%$ and $4 \%$ respectively. What can be observed from the plots is that the gain of CQI averaging is larger for medium to high vehicular speed $(30 \mathrm{~km} / \mathrm{h}$ to 60 $\mathrm{km} / \mathrm{h}$ ). This is because of a larger change in the channel conditions and hence received SIR over a period of $10 \mathrm{~ms}$. However for these speeds, the gain is more pronounced with channels providing very little or no multi-path diversity. In these channels the instantaneous received SIR varies quite rapidly and deeper fades can be observed. At very high speeds (such as $120 \mathrm{~km} / \mathrm{h}$ ) however, the gain is smaller as the rapid channel variation renders the averaging process incapable of tracking the SIR variation. Fig. 2 shows that limited gain is obtained for low speed $(3 \mathrm{~km} / \mathrm{h})$. At a low speed, the variation in channel and hence the received SIR is not rapid. Therefore, 
the received SIR does not vary significantly even after a period of $10 \mathrm{~ms}$. This is why small window sizes provide a small gain but higher window sizes results in performance loss (especially for channels that have no or negligible multipath diversity). This loss is due to a reduced ability to track the SIR variation. The curves for VA3 and PB3, two channels with significant multipath diversity, still show some gain even for higher averaging window sizes. This is because of the fact that multipath diversity mitigates the fading and hence the SIR variation.

Looking at all of the above results, it is clear that the primary factor that determines CQI averaging performance is the Doppler or the speed of mobile terminal. The other major factor, although with relatively less significant effect, is the multipath diversity of the fading channel.

\section{B. CQI Averaging Performance at Various Speeds}

To elaborate the performance of CQI averaging against terminal speed in isolation from multipath diversity, simulation results are presented in Fig. 3 for single-path Rayleigh fading channel at six different speeds $(3 \mathrm{~km} / \mathrm{h}, 10$ $\mathrm{km} / \mathrm{h}, 30 \mathrm{~km} / \mathrm{h}, 60 \mathrm{~km} / \mathrm{h}, 90 \mathrm{~km} / \mathrm{h}$ and $120 \mathrm{~km} / \mathrm{h}$ ). We see that gain in HSDPA throughput is small for small speeds but increases rapidly with increasing speed. Maximum gain is achieved at the medium speed of $30 \mathrm{~km} / \mathrm{h}$. However the gain starts to fall as the speed increases further. The peak gains in HSDPA throughput at various speeds are presented in Fig. 4; these peak gains are achieved against different averaging window sizes (as can be seen in Fig. 3). These results also support the discussion presented above.

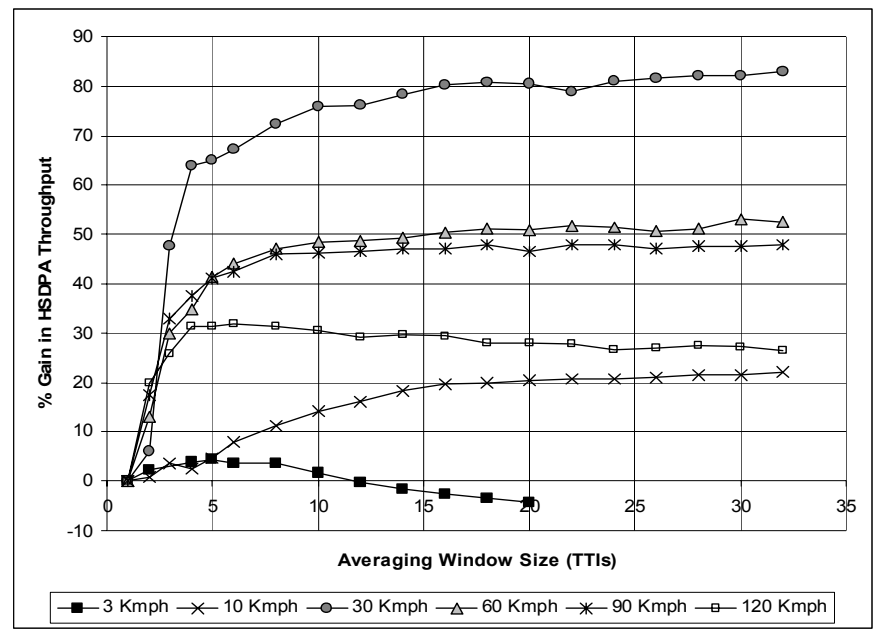

Figure 3: Effect of Speed on CQI Averaging Performance

\section{Selection of Suitable Window Size}

In order to obtain the greatest benefit from CQI averaging, the size of averaging window size needs to be adjusted according to radio channel conditions. It is possible to characterize the downlink radio channel with the help of CQI reports generated by the UE. Standard Deviation (STD) of CQI reports can be used to adjust the averaging window size to a suitable value. This suitable averaging window size is the minimum window size that provides an averaging gain equal to or close to the peak gain. Increasing window size beyond the suitable size, the CQI averaging gain either does not increase significantly or starts to decrease. The suitable window sizes for different fading channels are observed from the plots in Fig. 1 to Fig. 3 and presented in Table 2 along with the corresponding STD of CQI indices.

Since the dominant factor affecting the averaging gain and appropriate averaging window length is the UE speed, it is logical to look into the variation of STD of received CQI indices with UE speed. The suitable averaging window sizes for various mobile speeds in a single-path channel are plotted in Fig. 5 whereas Fig. 6 presents the corresponding STD of received CQI indices. STD is measured over a period of 1500 TTIs. The curves presented in Fig. 5 and Fig. 6 have very similar shape. This supports the use of STD of CQI indices as an appropriate measure to adjust averaging window size at Node-B transmitter.

Based on the observations for a wide variety of channels, we propose an adaptive approach to select the averaging window size at the Node-B that uses three different window sizes according to the measured STD of received CQI indices. In the beginning, a default window size of 5 can be selected as with this window size, reasonable performance gain is observed for nearly all the simulated channels. The window size can be adapted/refined as CQI reports are collected and STD measurements are taken. The optimization of window size adaptation rate and the STD measurement time interval are part of future work.

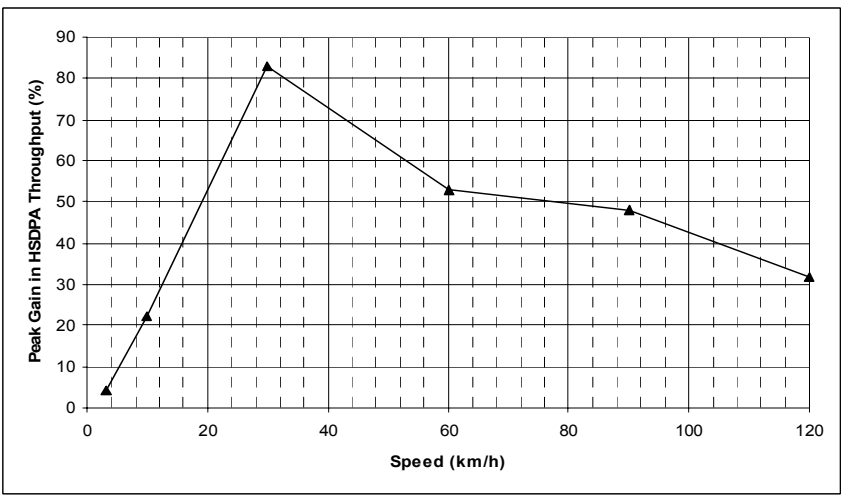

Figure 4: Peak Gain with Averaging at Different Speeds

Table 3 presents the proposed window sizes for different ranges of STD of received CQI indices: The default window size of 5 is to be used if the STD is less than 5. For STD values of 5 to 5.5 , a window size of 15 is to be used whereas for STD greater than 5.5, a window size of 20 is to be used. The algorithm in Table 3 is simple to implement yet achieves significant increase in HSDPA throughput. This can be observed by again looking at Fig. 1 to Fig. 3 and noting the gain in throughput for individual channels for the window sizes obtained from Table 3 against the STD values listed in Table 2. Gains in throughput corresponding to averaging window sizes derived from Table 3 are fairly close to the peak achievable gains for all the radio channels used in simulations 
TABLE II. STD OF RECEIVED CQI INDICES

\begin{tabular}{lll}
\hline Channel Profile & STD & Suitable Window Size \\
Single-Path $3 \mathrm{~km} / \mathrm{h}$ & 4.65 & 3 \\
Single-Path $10 \mathrm{~km} / \mathrm{h}$ & 5.66 & 18 \\
Single-Path $30 \mathrm{~km} / \mathrm{h}$ & 5.95 & 20 \\
Single-Path $60 \mathrm{~km} / \mathrm{h}$ & 5.30 & 14 \\
Single-Path $90 \mathrm{~km} / \mathrm{h}$ & 5.30 & 14 \\
Single-Path $120 \mathrm{~km} / \mathrm{h}$ & 5.20 & 4 \\
Pedestrian-B at $3 \mathrm{~km} / \mathrm{h}$ & 2.09 & 10 \\
Pedestrian-A at $3 \mathrm{~km} / \mathrm{h}$ & 4.21 & 5 \\
Pedestrian-A at $30 \mathrm{~km} / \mathrm{h}$ & 5.64 & 20 \\
Vehicular-A at $3 \mathrm{~km} / \mathrm{h}$ & 2.24 & 6 \\
Vehicular-A at $30 \mathrm{~km} / \mathrm{h}$ & 4.48 & 6 \\
Vehicular-A at $120 \mathrm{~km} / \mathrm{h}$ & 4.24 & 3
\end{tabular}

\section{TABLE III. PROPOSED CQI STD TO WINDOW SIZE MAPPING}

\begin{tabular}{ll}
\hline$S T D$ & Averaging Window Size \\
$<5$ & 5 (Default value) \\
$>5 \&<5.5$ & 15 \\
$>5.5$ & 20 \\
\hline
\end{tabular}

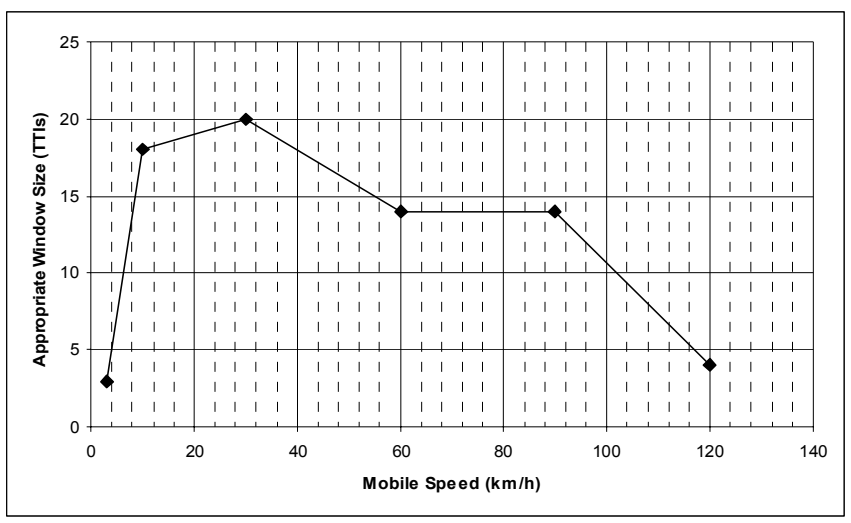

Figure 5: Appropriate Window Sizes at Different Speeds

\section{CONCLUSION}

A CQI averaging approach is proposed to improve the performance of link adaptation in HSDPA. In this approach, link adaptation is done at the Node-B transmitter on the basis of a moving average of SIR estimates derived from CQI indices reported by UE. It is shown for a variety of radio environments that CQI averaging provides significant gain in terms of link throughput without any change in terminal behaviour and only a small increase in complexity at Node-B. Suitable length of the moving average filter (averaging window size) is derived from the simulation results for different channels and mobile speeds. It is shown that the suitable window size is closely related to the standard deviation of the reported CQI indices. Finally, a three level adaptive approach is proposed to adjust the averaging window size on the basis of measured standard deviation of the CQI reports.

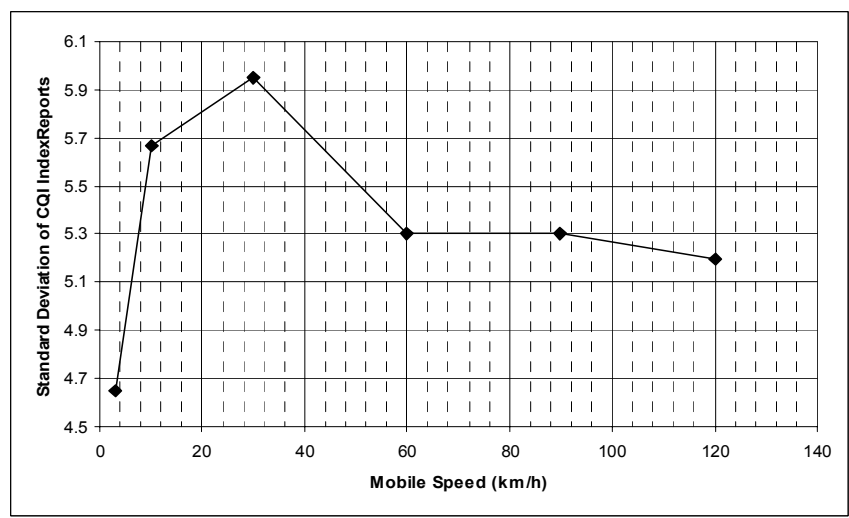

Figure 6: STD of Received CQI Indices at Different Speeds

\section{REFERENCES}

[1] H. Holma and A. Toskala, HSDPA/HSUPA for UMTS, $3^{\text {rd }}$ Edition. Wiley 2006.

[2] 3GPP Technical Specification 25.212, version 6.2.0, "Multiplexing and Channel Coding (FDD)", 06/2004.

[3] 3GPP Technical Specification 25.213, version 6.0.0, "Spreading and Modulation (FDD)", 01/2004.

[4] 3GPP Technical Specification 25.214, version 6.2.0, "Physical Layer Procedures (FDD)", 06/2004

[5] 3GPP document R1-02-0675 Motorola, Ericsson, "Revised CQI Proposal", 04/2002.

[6] F. Brouwer, I. de Bruin, J. C. Silva, N. Souto, F. Cercas and A. Correia, "Usage of Link-Level Performance Indicators for HSDPA NetworkLevel Simulations in E-UMTS", IEEE Eighth International Symposium on Spread Spectrum Techniques and Applications, Aug/Sep 2004.

[7] K. Ko, D. Lee. M. Lee and H. S. Lee, "A Novel SIR to Channel-Quality Indicator (CQI) Mapping Method for HSDPA System", IEEE 64th Vehicular Technology Conference (VTC), Fall 2006.

[8] K. Freudenthaler, A. Springer, and J. Wehinger, "Novel SINR-to-CQI Mapping Maximizing the Throughput in HSDPA", IEEE Wireless Communications and Networking Conference (WCNC), Mar 2007.

[9] 3GPP Technical Report 25.858, version 5.0.0, "High Speed Downlink Packet Access: Physical Layer Aspects", 03/2002.

[10] D. Martin-Sacristan, J. F. Monserrat, J. Gozalvez and N. Cardona, "Effect of Channel-Quality Indicator Delay on HSDPA Performance", IEEE 65th Vehicular Technology Conference (VTC), Spring 2007.

[11] 3GPP Technical Specification 25.211, version 6.7.0, "Physical Channels and Mapping of Transport Channels onto Physical Channels (FDD)", $12 / 2005$.

[12] 3GPP Technical Specification 25.101, version 6.4.0, "User Equipment (UE) Radio Transmission and Reception (FDD)", 03/2004.

[13] S. Kim, Y. Lee and H. Choi, "Velocity Estimation using WCDMA Uplink Signal for Adaptive Modulation and Coding in HSDPA System", The 9th Asia-Pacific Conference on Communications (APCC), Sep 2003.

[14] J. Kim, Y. Hong and D. K. Sung, "A Radio Channel Estimation Scheme Using the CQI Feedback Information in High Speed Downlink Packet Access", IEEE International Conference on Communications (ICC), Jun 2006.

[15] A. U. Quddus and R. Tafazolli, "SIR Estimation on Common Pilot Channel with the Knowledge of Data to Pilot Power Ratio for Closed Loop Power Control in WCDMA FDD Downlink", IEEE 60th Vehicular Technology Conference (VTC), Fall 2004. 